\title{
Intensidad exportadora y fortalezas percibidas en la estrategia de marketing: Una perspectiva basada en la experiencia de empresas exportadoras españolas
}

\author{
Pilar Castro-González(D), Belén Gutiérrez-Villar(iD), Rafael Ángel Araque-Padilla(iD, \\ María José Montero-SimóiD \\ Universidad Loyola Andalucia (Spain)
}

pcastro@uloyola.es,_belengut@uloyola.es,raraque@uloyola.es,jmontero@uloyola.es

Received April, 2016

Accepted October, 2016

\section{Resumen}

Objeto: Estudiar si existe asociación entre el perfil exportador de una muestra de empresas españolas -alta o baja intensidad exportadora- y la opinión de sus responsables sobre las fortalezas generadas en las variables del mix de marketing.

Diseño/metodología/enfoque: A través de un cuestionario a 64 empresas exportadoras españolas y en base a la revisión de la literatura existente, definimos como variable dependiente la intensidad exportadora. Las variables explicativas miden la importancia otorgada a las diferentes variables del mix de marketing en tanto que fortalezas reconocidas para la actividad exportadora. Se aplica el método MANOVA, ya que nos permite analizar las diferencias de dos o más variables métricas dependientes (calidad, precio, comunicación y distribución) basadas en un conjunto de variables categóricas que actúan como predictoras (volumen exportador).

Aportaciones y resultados: Los resultados nos permiten apoyar la idea de que una correcta planificación de las estrategias de marketing supone un aspecto clave dentro de la estrategia de internacionalización de las empresas. Frente a otros estudios que analizan estrategias específicas dentro de las variables del marketing mix, este trabajo se centra en la consideración de dichas 
variables en tanto que fortalezas competitivas percibidas por las empresas en su actividad exportadora, sea cual sea la estrategia específica, dentro de la variable del mix, que genere dichas fortalezas.

Implicaciones prácticas: Las diferencias observadas indican que existen patrones diferentes en la evaluación de las fortalezas en el mix de marketing. Así, los resultados apuntan a que las empresas más exportadoras han generado mayores capacidades principalmente en dos variables del mix de marketing: la configuración de sus canales de distribución, así como en sus estrategias de comunicación.

Originalidad / Valor añadido: El principal elemento diferenciador del estudio es el enfoque conjunto de las variables del mix de marketing como fortalezas competitivas, pero distinguidas una a una.

Palabras clave: Internacionalización, Recursos y capacidades, Criterios competitivos, MANOVA, Marketing

Códigos JEL: F2, M310

Title: Export intensity and strengths perceived in the marketing strategy: A perspective based on Spanish export companies' experience

\section{Abstract}

Purpose: This paper studies if there are associations between export profiles of some Spanish companies -high or low export intensity- and the opinion of managers about strengths generated in the marketing mix.

Design/methodology: Through a questionnaire to 64 export Spanish companies and the literature review, we defined the export intensity as the dependent variable. The explanatory variables used measure the importance granted by the companies to the different variables of the marketing mix as strengths for export activity. We used MANOVA method as it lets us analyze differences between two or more metrical dependent variables (quality, price, promotion and place) based on a mix of categorical variables that act like predictors (export volume).

Findings: Our results indicate that a correct planning of the marketing strategy is a key aspect inside internationalization strategy. In contrast with other studies that analyze specific strategies 
within marketing mix, this research focuses on these as perceived competitive strengths, whichever the mix of variable strategy that generates these strengths is.

Practical implications: The differences observed indicate that there are different patterns in the marketing mix strength evaluation. The results show that the firms which have the most export intensity have generated bigger capacities especially in distribution channels configuration and promotion strategies.

Originality/value: The main differentiating element in this research is the combines focus on the marketing mix variables as competitive strengths while distinguishing each of them.

Keywords: Internationalization, Resources and capabilities, Competitive advantage, MANOVA, Marketing

Jel Codes: F2, M310

\section{Introducción}

Numerosos estudios confirman que una creciente presencia en los mercados internacionales permite ganar dimensión y limitar los riesgos-país asociados a la actividad. La entrada en mercados extranjeros supone la generación de nuevas oportunidades de negocio (Sapienza, Autio, George \& Zahra, 2006). En buena parte reforzado por la situación actual, la internacionalización supone una importante estrategia para asegurar la supervivencia de la empresa en el largo plazo (Parada, 2012). De hecho, cada día son más las empresas que exportan sus productos tal y como indican los datos de la balanza comercial española, que arroja records históricos, con el primer superávit desde 1971.

La internacionalización de las empresas es una estrategia importante para el desarrollo económico y una alternativa muy a tener en cuenta para su crecimiento y competitividad. Por este motivo, se ha incentivado una intensa labor investigadora que ha tratado de explicar el fenómeno, dando lugar a un cuerpo abundante de teorías sobre internacionalización (Johanson \& Wiedersheim-Paul, 1975; Bilkey \& Tesar, 1977; Johanson \& Vahlne, 1977, 1990; Dunning, 1988, 2001; Andersson, 2000; Rialp, Rialp \& Knight, 2005). A pesar de las numerosas contribuciones tanto teóricas como metodológicas, ninguna de ellas parece explicar de forma completa la complejidad del fenómeno de la internacionalización de las empresas (Liberman, Baena \& Moreno, 2010). Tampoco parece existir consenso sobre cuáles son los 
determinantes que afectan a los resultados de las empresas exportadoras (Losada, Navarro, Ruzo \& Barreiro, 2006).

Partiendo de una extensa revisión de la literatura científica publicada entre 1998 y 2005 en las revistas de marketing e internacionalización más reconocidas Sousa, Martínez-López y Coelho (2008) identifican y examinan factores externos e internos de influencia en la actuación exportadora. Dicha clasificación se basa en dos enfoques teóricos usuales: la teoría de los recursos y capacidades y el enfoque de la contingencia. Bajo el primero de los enfoques, se asume que la acumulación de recursos puede otorgar a la empresa la capacidad suficiente para internacionalizarse (Wernerfelt, 1984; EscuderoTorres, Hurtado-Torres, de la Torre-Ruiz \& Aguilera-Caracuel, 2011; Rodríguez, Ruiz \& Martín Armario, 2012). Los recursos y capacidades influyen en la elección de la estrategia competitiva y en la obtención de ventajas en mercados exteriores (Morgan, Kaleka \& Katsikeas, 2004), y la percepción de dichas ventajas competitivas es uno de los motivos para comenzar el proceso de internacionalización (Cavusgil \& Nevin, 1981). En contraste, el paradigma de la contingencia sugiere que los factores del entorno influyen de manera determinante en la estrategia de la empresa y en su comportamiento exportador, de tal modo que la influencia de los factores internos está condicionada por factores externos (Yeoh \& Jeong, 1995; Robertson \& Chetty, 2000).

Entre los factores internos más analizados como antecedentes del comportamiento exportador destaca la estrategia de marketing, siendo la variable producto el aspecto que más atención suscita, seguido del precio, la comunicación y la distribución.

Generalmente, los estudios que analizan la influencia de esas variables de marketing suelen basarse en la conveniencia de su adaptación o estandarización a los mercados exteriores, pero es menos frecuente analizar en qué medida dichas variables son percibidas por las empresas como recursos clave en su proceso de internacionalización. Puesto de otro modo, cabe cuestionarse si las empresas, en función de su intensidad exportadora, se reconocen diferentes fortalezas competitivas en su estrategia de marketing. Análisis de este cariz nos permitiría avanzar en el conocimiento de las estructuras clave que favorecen el proceso de internalización. Justamente, en este trabajo nos proponemos como objetivo estudiar si existe una asociación entre el perfil exportador de las empresas y las fortalezas generadas en las variables del marketing mix.

El trabajo se estructura de la siguiente forma. Primero se justifican las hipótesis de estudio a la luz de la revisión teórica realizada. En segundo lugar, se expone el diseño de la investigación y la metodología empleada, para terminar con los resultados y conclusiones derivadas del estudio. 


\section{Objeto de investigación e hipótesis de estudio}

Como se indicó previamente, el objeto de esta investigación es estudiar si existe asociación entre el perfil exportador de una muestra de empresas españolas -alta o baja intensidad exportadora- y la opinión de sus responsables empresariales sobre las fortalezas generadas en las variables del mix de marketing. Dado dicho objetivo, en los párrafos siguientes se van a justificar las diferentes hipótesis de estudio que nos permitan, por un lado, contrastar la existencia de diferencias en las variables de marketing simultáneamente, según el perfil exportador de la empresa, y por otro, la existencia de diferencias variable a variable del marketing mix.

H1: El perfil exportador de las empresas va asociado a la existencia conjunta de fortalezas competitivas en las variables del marketing mix.

Son diversos los autores que han analizado la relación de la estrategia de marketing, medida a través de diferentes variables del mix, con el perfil exportador de las empresas. Una de las primeras investigaciones que indagaba sobre esta relación fue la de Cavusgil (1983) donde se identificaba cuatro grupos de variables significativas en la estrategia de marketing: oferta de la empresa en términos de calidad, servicio post-venta, el papel de los distribuidores, las actividades de promoción y la variable precio. Estos resultados fueron avalados y ampliados por autores como Moini (1995), Kaleka y Katsikeas (1995), Katsikeas, Piercy y Ioannidis (1996), Zou, Fang y Zhao (2003), Morgan, Vorhies y Mason (2009) y Morgan, Katsikeas y Vorhies (2012).

En España se han hecho también estudios en la misma línea como es el caso de Gómez y Valenzuela (2005) donde a partir de una muestra de empresas exportadoras "activas" españolas, demostraron que los diferentes niveles de actuación exportadora están relacionados con los diferentes elementos de la estrategia de marketing tales como la adaptación del producto, el nivel de precios, la adaptación de la marca y la elección de los canales de distribución, siendo esta última la variable más explicativa. En la misma idea inciden Pla-Barber, Villar-García y Escribá Esteve (2010), Martínez-Villavicencio (2011), Navarro, Acedo, Losada y Ruzo (2011) y Fernández Moreno, Peña García-Pardo y Hernández Perlines (2007).

Por tanto, los estudios pueden llevar a pensar que el desarrollo de fortalezas competitivas en la estrategia combinada del mix de marketing puede asociarse con el perfil exportador de la empresa, tal y como se refleja en nuestra hipótesis primera. 
H2: No todas las variables del mix (calidad del producto, precio, comunicación y distribución) presentan diferencias entre las empresas con alta intensidad exportadora y el resto de empresas exportadoras.

Esta hipótesis se ha desagregado en cuatro, una por variable, cuyo sustento teórico se expone a continuación.

H2.1: La importancia otorgada a la calidad como fortaleza competitiva en las empresas no establece diferencias en su perfil exportador.

Como se dijo anteriormente, la variable producto es la variable más estudiada en relación con el comportamiento exportador de la empresa (Piercy, Kaleka \& Katsikeas, 1998; Brouthers \& Xu, 2002; Morgan et al., 2004; Brouthers \& Nakos, 2005). Más específicamente, se ha puesto de manifiesto la especial relevancia de la calidad por su influencia en el proceso de internacionalización (Rastrollo, Martín \& González, 2014). De ahí que en nuestro estudio nos hayamos centrado en la calidad como medida de la variable producto.

En este sentido, no son coincidentes las conclusiones obtenidas en los estudios revisados. Así, es posible encontrar investigaciones que apuntan a una relación positiva entre calidad del producto y el rendimiento internacional de las empresas exportadoras (Calantone \& Knight, 2000). Autores como Villena y Souto (2015) concluyeron que la certificación de calidad impactaba de manera positiva en el desempeño exportador de una muestra de empresas españolas. Por el contrario, y en el caso de empresas españolas, las conclusiones más recientes parecen apuntar en el sentido de la ausencia de relación. En esta línea encontramos trabajos (Flor \& Oltra, 2010; Estrella, Jiménez, Ruiz \& Sánchez, 2012) donde se afirma que, para competir con éxito en mercados internacionales, la calidad de los productos es un elemento necesario, pero no suficiente, no debiendo ser entendida como un valor añadido, sino como un requisito sine qua non para competir en los mercados. Por tanto, es previsible que, aun siendo un aspecto competitivo importante para las empresas exportadoras españolas, la calidad no sea el elemento más diferenciador para distinguir entre perfiles exportadores.

H2.2: La importancia otorgada al precio de los productos como fortaleza competitiva en las empresas no establece diferencias en su perfil exportador.

En relación con dicha estrategia, la literatura no es contundente ya que se trata de un campo aún por explorar con mayor rigor y consistencia (Tan \& Sousa, 2011). La mayoría de los estudios se centran en el análisis de la influencia de la estandarización de precios sobre la actuación exportadora, llegándose a resultados contradictorios (Zou, Andrus \& Norvell, 1997; Lages, Abrantes \& Lages, 2008; Sousa \& 
Lengler, 2009; Navarro, Acedo, Robson, Ruzo \& Losada, 2010; Navarro et al., 2011; MartínezVillavicencio, 2011). Así, cabe cuestionarse si en general el precio es una fortaleza relevante para la actuación exportadora o como afirman Cavusgil y Zou (1994) nos encontramos ante una variable irrelevante para la construcción de una ventaja competitiva en el proceso de internacionalización de las empresas.

H2.3: La importancia otorgada a la variable comunicación como fortaleza competitiva establece diferencias en el perfil exportador.

La actuación exportadora de la empresa también se ha relacionado con la estrategia de comunicación en numerosos estudios (Gómez \& Valenzuela, 2005; Sousa et al., 2008; Estrella et al., 2012). La mayoría de ellos ponen de manifiesto que las empresas con mayor perfil exportador obtuvieron una mayor competitividad internacional a través de sus procesos de comunicación.

H2.4: La importancia otorgada a la distribución como fortaleza competitiva establece diferencias en el perfil exportador.

Son diversos los estudios que ponen de manifiesto la relación entre la variable distribución y el perfil exportador de las empresas (Alonso \& Donoso, 2000; García-Canal, 2004; Gómez \& Valenzuela, 2005; Aragón \& Monreal, 2008; Navarro et al., 2011; Estrella et al., 2012). La mayoría de estos estudios se enfocan en el análisis de la influencia de estrategias concretas de distribución (ej: canales de distribución propios), poniendo de manifiesto una relación con la actuación exportadora condicionada a otros factores externos u organizativos. En este estudio partimos de la base de la percepción que tiene la empresa sobre su nivel competitivo en la variable distribución, sin entrar al detalle de estrategias específicas al albur de otro tipo de factores condicionantes. 


\section{Diseño de la investigación y metodología}

\subsection{Captación de la información}

El presente trabajo se nutre de parte de la información captada en una investigación más amplia diseñada y emprendida con otras universidades europeas. Los principales resultados del estudio internacional mencionado, así como el cuestionario global utilizado pueden consultarse en el libro European Studies on Intercultural dimension of International Business (Horská et al., 2011). En este trabajo sólo se han utilizado algunas de las preguntas y respuestas recopiladas sobre datos de exportación y valoraciones de las variables de marketing correspondientes a las empresas exportadoras españolas de la muestra.

Para la selección de la muestra española del estudio se recurrió a la base de datos "España 25,000" (2007), editada por Fomento de la Producción, S.L. La base de datos contiene los principales datos actualizados de 25,000 compañías españolas, tales como señas postales, web, correo electrónico y cargos directivos. También detalla la actividad de cada empresa, participación extranjera en su capital, filiales dentro y fuera de España, principales accionistas y en algunos casos, volumen de producción y localización de las factorías. De las 25,000 empresas aparecían registradas 780 empresas exportadoras, de las cuales se eliminaron aquellas con capital extranjero, quedando finalmente un total de 630 empresas. La recogida de información se efectuó mediante el uso de un cuestionario autoadministrado y anonimizado. Los mismos se enviaron por correo electrónico hasta en tres ocasiones; al mismo tiempo, se hizo un envío postal adicional y se apoyó con llamadas telefónicas solicitando la cumplimentación del cuestionario. Todo ese proceso de captación de información implicó nueve meses comprendidos entre junio de 2008 y febrero de 2009.

De las 630 empresas a las que se les envió el cuestionario, siguiendo las distintas vías, vinieron devueltos 33 cuestionarios por no ser correcta la dirección de envío. De las restantes, se recepcionaron correctamente cumplimentados 64 cuestionarios, lo que representa un 10.7\% de tasa de respuesta. 


\subsection{Descripción de las variables}

En nuestro estudio, la variable a explicar “perfil exportador o desempeño exportador"la medimos a través de una pregunta relativa a la intensidad exportadora (porcentaje de ventas, sobre ventas totales de cada empresa), tal y como se lleva a cabo en numerosos estudios (Dhanaraj \& Beamish, 2003; Morgan et al., 2004; Claver, Rienda \& Quer, 2006; Flor \& Oltra, 2010). Inicialmente en el cuestionario se optó por solicitar esta información para tres ejercicios económicos consecutivos y se proporcionó a los encuestados una escala con 7 intervalos de valores.

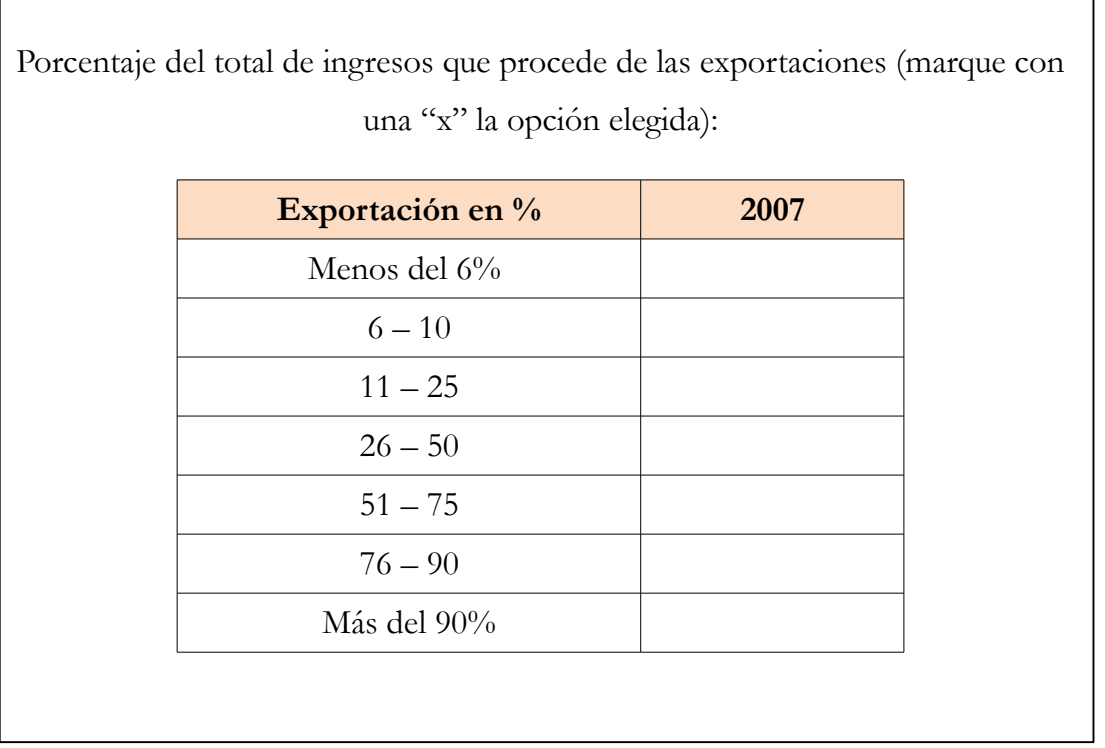

Tabla 1. Pregunta utilizada para medir la variable a explicar "perfil exportador"

Las respuestas obtenidas para cada empresa en los tres años indagados no presentaban diferencias estadísticamente significativas (el intervalo seleccionado por cada empresa se mantiene constante prácticamente año a año) por lo que se manejaron únicamente las cifras correspondientes al año 2007. Tal y como se observa en la Tabla 1, se proporcionó a los encuestados una escala con 7 intervalos de valores; sin embargo, para el objeto de este análisis y dado el reducido tamaño de la muestra se han reducido a dos las posibilidades, quedando por tanto la categorización de la empresas reducida a 2 grupos: empresas con alta intensidad exportadora (es decir, aquellas cuyas ventas en mercados distintos al nacional es superior o igual al 75\%) y resto de empresas exportadoras (cuyo porcentaje de ventas en mercados extranjeros no llega al 75\%), dicotomización que se considera adecuada para el número de observaciones manejadas (64 empresas). Un procedimiento de agrupación de la variable intensidad 
exportadora es empleado por Graves y Thomas (2006), distinguiendo entre empresas locales o no exportadoras, empresas moderadamente exportadoras (si se encuentran por debajo de la mediana de la ratio) y empresas intensamente exportadoras (si se encuentran por encima de la mediana del ratio). Moini (1995) la emplea conjuntamente con el crecimiento de las exportaciones como variable dependiente. De esta forma, establece tres grupos de empresas en función del valor de sendas variables.

La operativización de las variables explicativas - las cuatro pes del mix- se ha basado en la importancia otorgada por las empresas a las diferentes variables del mix de marketing en tanto que fortalezas reconocidas para la actividad exportadora. Es decir, se ha partido de la percepción que tiene el personal directivo respecto de las posibles ventajas competitivas en el ámbito de marketing generadas por su propia empresa. Esta manera de proceder tiene precedentes en estudios sobre el perfil de las empresas exportadoras. Así, autores como Reuber y Fischer (1998) defienden que las decisiones asociadas al proceso de internacionalización dependen, en última instancia, de las motivaciones y habilidades de los directivos implicados en el proceso. Según Leonidou, Katsikeas y Piercy (1998), las aptitudes y actitudes de los directivos, como la confianza que ellos tengan en el éxito de la estrategia exterior, resultan determinantes a la hora de explotar las capacidades competitivas de la empresa en los mercados internacionales.

La evaluación de estas percepciones sobre el grado de fortaleza competitiva se ha hecho a partir de las puntuaciones otorgadas en una escala de 1 a 10 (ver Tabla 2) para cada una de las variables, valoradas independientemente unas de otras, de acuerdo a las consideraciones derivadas de la revisión de la literatura expuesta en los apartados precedentes y reflejadas en las hipótesis de este trabajo.

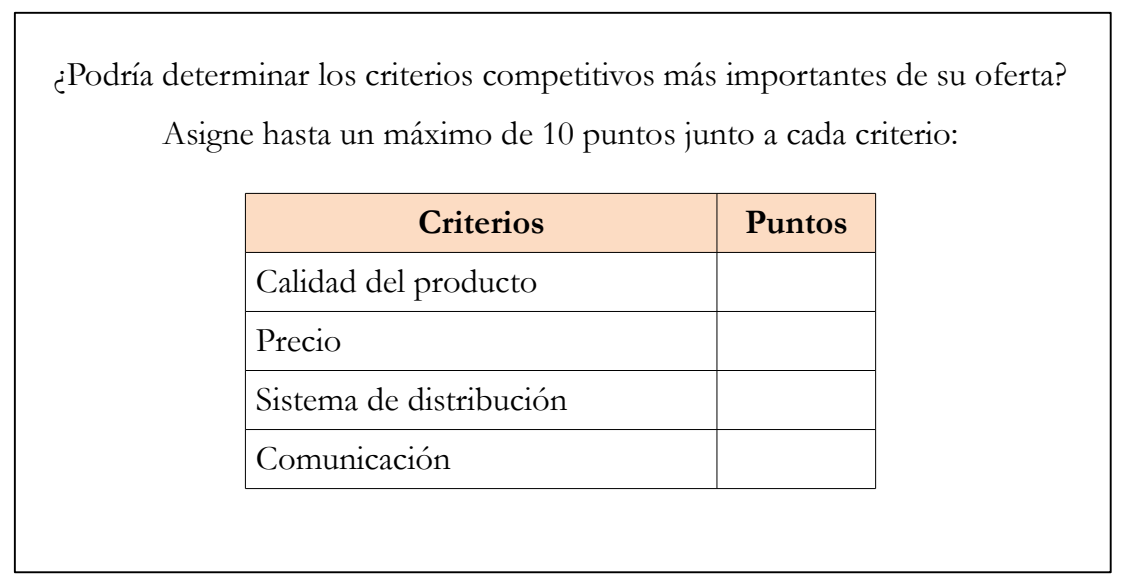

Tabla 2. Pregunta propuesta para medir la fortaleza competitiva en las variables del mix de marketing 


\subsection{Metodología}

Una de las técnicas estadísticas más apropiadas para analizar las diferencias de dos o más variables métricas dependientes (en este caso, calidad del producto, precio, comunicación y sistema de distribución) basadas en un conjunto de variables categóricas que actúan como predictoras (en nuestro caso, el volumen exportador) es el análisis multivariante de la varianza-MANOVA- (Hair, Anderson, Tatham \& Black, 1999). Podría haberse recurrido como alternativa al MANOVA al uso de la T2 de Hotelling al tratarse de una variable independiente con dos niveles. Sin embargo, se ha usado el MANOVA cuyos resultados son equivalentes por no disponer SPSS de esta prueba. La técnica MANOVA proporciona dos tipos de resultados:

- Multivariados, con los que se evalúan las posibles diferencias existentes entre grupos para las variables endógenas consideradas simultáneamente, es decir, el efecto del factor (variable explicativa) sobre el conjunto de las variables endógenas;

- Univariados, con los que se miden las diferencias variable a variable, siempre y cuando se haya contrastado que existen globalmente.

Con ello se trata de determinar en qué variables se producen las diferencias entre grupos.

Ambos tipos de resultados serán la base para la contrastación de las hipótesis planteadas en esta investigación: la prueba global es adecuada para el contraste de la Hipótesis 1, pues en ella se evalúan simultáneamente las diferencias entre grupos para todas las variables dependientes, mientras que las pruebas univariadas (denominadas efectos inter-sujetos en los resultados MANOVA) se han utilizado para contrastar variable a variable, las posibles diferencias entre grupos, lo que se corresponde con la verificación de las hipótesis dos a cinco. 


\section{Resultados}

Para la contrastación de la Hipótesis 1 se ha recurrido al análisis MANOVA. La correcta aplicación de MANOVA requiere estudiar, previamente, un conjunto de condiciones sobre el comportamiento de las variables endógenas utilizadas. La normalidad de las variables endógenas, es la primera de las condiciones necesarias. En nuestro caso, la prueba de normalidad de Kolmogorov-Smirnov aplicada a cada una de las cuatro variables de nuestro estudio arroja el siguiente resultado:

\begin{tabular}{|l|l|}
\hline Variable & Significación(a) \\
\hline Calidad & 0.003 \\
\hline Precio & 0.178 \\
\hline Distribución & 0.092 \\
\hline Comunicación & 0.318 \\
\hline
\end{tabular}

(a)Corrección de la significación de Lilliefors.

Tabla 3. Prueba de normalidad de Kolmogorov-Smirnov para las variables dependientes

A la vista de estos resultados, con un nivel de significación del 5\%, podemos aceptar la normalidad de todas las variables salvo para el caso de la calidad del producto.

Los intentos de transformar esta variable con objeto de normalizarla (se han probado las transformaciones logarítmica y la potencial con distintos exponentes) no han mejorado las significaciones de manera apreciable, por lo que se ha optado por no someter la variable a ninguna transformación, utilizando sus valores originales. Dada la relativa robustez de la prueba MANOVA y puesto que sólo una de las cuatro variables endógenas no se ajusta a la distribución normal, se ha considerado aceptable la inclusión de la variable calidad en los análisis, dado que las otras variables endógenas sí lo son. Otra condición que deben cumplir las variables analizadas para la aplicación de la técnica MANOVA es la homogeneidad de las matrices de varianza-covarianza por grupos. Se ha recurrido a la prueba de Box obteniéndose una significación $=0.336$, por lo que se cumple el requisito de homogeneidad, es decir, podemos aceptar el supuesto de que las matrices de varianza-covarianza de las variables dependientes son iguales en los dos grupos determinados por la independiente.

Otra característica que se debe analizar antes de elaborar un MANOVA guarda relación con la condición de multicolinealidad entre las variables endógenas; así, debería existir cierto grado de correlación entre ellas, pero no excesivo. Se han realizado análisis de correlaciones paramétricos y no paramétricos, para estudiar dicho aspecto entre las variables dependientes, apareciendo una correlación significativa pero escasa. El valor máximo es de 0.389 lo que sugiere que no existen problemas de 
multicolinealidad entre las variables analizadas, siendo aptas para este tipo de análisis. Se ha optado por la construcción de un modelo MANOVA a través del origen -sin término independiente- Hair et al. (1999, pp. 392).

Para contrastar la igualdad conjunta de valores medios en ambos grupos de empresas (hipótesis nula del MANOVA) se emplean habitualmente cuatro estadísticos de contraste (raíz mayor de Roy, traza de Hotelling, Lambda de Wilks y traza de Pillai). La utilización de estas cuatro pruebas, en caso de conducir a la misma conclusión, incrementa su fiabilidad y proporciona más evidencia que si sólo se recurre a una de ellas. En nuestro caso, la tabla 4 muestra que todos los estadísticos aplicados alcanzan valores elevados y además con una significación prácticamente nula (sig $=0.000$ para los cuatro estadísticos). Como consecuencia, se rechaza la hipótesis nula del modelo MANOVA, por lo que no puede aceptarse que el valor medio del conjunto de las variables analizadas sea el mismo para ambos grupos (empresas más o menos exportadoras).

\begin{tabular}{|l|r|r|r|r|r|r|}
\hline Estadístico & \multicolumn{1}{|l|}{ Valor } & F & gl. Hipótesis & gl. error & Sig. & Potencia \\
\hline Traza de Pillai & 1.203 & 10.569 & 8 & 56 & 0.000 & 1,000 \\
\hline Lambda de Wilks & 0.006 & 80.569 & 8 & 54 & 0.000 & 1,000 \\
\hline Traza de Hotelling & 131.349 & 426.884 & 8 & 52 & 0.000 & 1,000 \\
\hline Raíz mayor de Roy & 131.082 & 917.573 & 4 & 28 & 0.000 & 1,000 \\
\hline
\end{tabular}

Tabla 4. Contrastes multivariados para MANOVA

Esto indica, a efectos de nuestra investigación, que las diferencias en capacidad exportadora se relacionan con diferencias en las percepciones sobre la importancia que otorgan al mix de marketing (aceptación de la Hipótesis H1).

A continuación se presentan los análisis necesarios para contratar las hipótesis restantes. Como se explicó en la metodología se ha recurrido a las pruebas inter-sujetos. La identificación de las variables endógenas en las que se producen las diferencias requiere de los contrastes individuales para cada una de ellas, que a su vez están supeditados a la verificación de la igualdad de las varianzas residuales. Para comprobar este supuesto se ha recurrido al contraste de Levene cuyos valores han conducido a la aceptación de esta condición previa.

En la Tabla 5 se muestran las citadas pruebas inter-sujetos. Los valores de las significaciones (sig. $=0.000)$ permiten afirmar que existen diferencias significativas en los valores medios de cada una de las cuatro variables investigadas, relacionadas con el grupo a que las empresas pertenecen según su nivel de exportación. Por tanto, se confirman la Hipótesis 2.3 y la Hipótesis 2.4., rechazándose las Hipótesis 
2.1. y 2.2. Esto quiere decir que, tal y como se suponía, según las investigaciones revisadas, la comunicación y la distribución son diferentemente valoradas por las empresas exportadoras. Por otra parte, y en contra de las hipótesis planteadas, la calidad del producto y el precio también han demostrado tener una valoración diferente en ambos grupos de empresas.

\begin{tabular}{|l|r|r|r|r|r|}
\hline Variable & Suma de cuadrados Tipo III & Gl. & Media Cuadrática & F & Sig. \\
\hline Calidad & 2757.009 & 2 & 1378.505 & 1723.784 & 0.000 \\
\hline Precio & 1892.545 & 2 & 946.273 & 461.938 & 0.000 \\
\hline Distribución & 1913.282 & 2 & 956.641 & 956.641 & 0.000 \\
\hline Comunicación & 1850.627 & 2 & 925.314 & 925.314 & 0.000 \\
\hline
\end{tabular}

Tabla 5. Pruebas de los efectos inter-sujetos

$\mathrm{Si}$ analizamos variable a variable los resultados (Tabla 5), sabiendo que todas las diferencias entre medias son significativas y atendiendo a los valores parciales de la $\mathrm{F}$, teniendo en cuenta que valores de la F grandes indican una capacidad discriminante mayor (Hair et al. 1999, pp. 276), las conclusiones son las siguientes a la vista de los valores del citado estadístico: las mayores diferencias entre ambos tipos de empresas exportadoras se producen en la variable calidad ( $\mathrm{F}=1,723.784)$, seguida de la distribución y la comunicación, con valores del estadístico muy similares. Finalmente, y con un efecto mucho menor en la diferenciación de grupos, se encuentra la importancia otorgada al precio.

\section{Conclusiones}

Los resultados alcanzados en el presente estudio nos permiten apoyar la idea de que una correcta planificación de las estrategias de marketing supone un aspecto clave dentro de la estrategia de internacionalización de las empresas. El objetivo que nos planteábamos era estudiar si puede establecerse una asociación entre fortalezas competitivas percibidas en dicha estrategia y el perfil exportador (medido en términos de intensidad exportadora), para lo cual se formularon una serie de hipótesis.

Como primera hipótesis se postulaba que diferencias entre perfiles exportadores se asocian, más allá de efectos individuales, a patrones combinados de fortalezas competitivas en las variables del mix de marketing. La corroboración de esta hipótesis nos permite colegir que la actuación exportadora no tiene por qué favorecerse solo generando fortalezas competitivas aisladamente en alguna de las variables claves de marketing, sino que tiene relevancia el conjunto combinado de fortalezas. Puesto de otra 
forma, las diferencias observadas indican que existen patrones diferentes de fortalezas en el mix de marketing entre las empresas más o menos exportadoras.

Por la naturaleza de los análisis que se han hecho, no podemos cuantificar el papel que cada una de las variables tiene en dicho patrón, lo que resultaría de interés para establecer prioridades de mejora, pero sí nos permite subrayar la idea de la necesidad de analizar el conjunto, y no solo de realizar, como por otro lado es frecuente, estudios parcelados sobre influencias individuales de variables. En otras palabras, la concreción en cada empresa de cada una de las variables del mix en estrategias o tácticas específicas y adaptadas a cada sector y situación no debería hacerse sin tener en cuenta la coherencia y fortalezas en el resto de variables.

Por otro lado, asumido ese efecto conjunto, se han hecho análisis variable a variable para ver cómo se concretan las diferencias entre patrones según intensidad exportadora. Con relación a la calidad (variable producto), se han obtenido diferencias significativas entre el grupo de empresas más y menos exportadoras, por lo que la importancia competitiva otorgada por las empresas difiere entre ambos grupos, y es además la que más los diferencia, es decir, aun siendo las tres variables restantes también significativas, la calidad del producto es la mejor identificadora de las empresas de cada grupo, por la homogeneidad de valoraciones sobre este aspecto de marketing en cada categoría. Por lo tanto, no es posible mantener la Hipótesis 2.1. Así, parece que, en el sentido de otras investigaciones referenciadas en nuestra investigación sobre los resultados en empresas de otras nacionalidades, la generación de fortalezas competitivas en calidad es una característica claramente diferenciadora en los resultados de la exportación. Respecto del precio, los resultados obtenidos nos han conducido a rechazar la Hipótesis 2.2, en la que se planteaba que dicha variable no establece diferencias entre grupos de empresas. Sería interesante analizar con más detalle en futuros estudios el significado de esa competitividad en precios, dado que la importancia de dicha variable puede asociarse, por ejemplo, a la generación de capacidades como estructuras competitivas de costes, o bien a la relación de esta variable con otros aspectos como la calidad o el servicio al cliente (Martínez-Villavicencio, 2011). Igual sucede con las variables de distribución y comunicación. Lo encontrado en este estudio apunta hacia lo que se había esperado, es decir, la diferente importancia otorgada según el perfil exportador (Hipótesis 2.3 y 2.4). Estos resultados pueden indicar que las empresas de mayor intensidad exportadora han desarrollado especialmente mayores capacidades, tanto en la configuración de sus canales de distribución, como en sus estrategias de comunicación.

En suma, los resultados anteriores nos permiten afirmar la existencia de una asociación entre las variables del mix de marketing y el perfil exportador de las empresas. Si bien, frente a otros estudios 
que analizan estrategias específicas dentro de las variables del marketing mix, este trabajo se centra en la consideración de dichas variables en tanto que fortalezas competitivas percibidas por las empresas en su actividad exportadora, sea cual sea la estrategia específica, dentro de la variable del mix, que genere dichas fortalezas. Otro elemento diferenciador del estudio es el enfoque de análisis de las variables del marketing mix como fortalezas competitivas dejando de lado el tan habitual análisis sobre la idoneidad de la adaptación o estandarización de dichas variables en los diferentes mercados.

Nuestra investigación presenta algunas limitaciones, entre las que cabe destacar el reducido tamaño de la muestra, dada la baja tasa de respuesta obtenida. Así, el reducido tamaño muestral ha limitado las técnicas de análisis que pueden emplearse: MANOVA es factible (tal y como se expuesto en los apartados precedentes), pero no hemos podido aplicar técnicas predictivas que requieren muestras de mayor tamaño. Así, con una muestra de mayor tamaño habría sido posible aplicar además de MANOVA, otros procedimientos que permitiesen no solo detectar efectos sino también cuantificarlos: la regresión logística, por ejemplo.

Como líneas de investigación futuras sería interesante profundizar en las estrategias de marketing llevadas a cabo por las empresas con una mayor intensidad exportadora en aras de construir un modelo para intentar afrontar con éxito la internacionalización de las empresas españolas. Éste puede resultar de utilidad para el alto porcentaje de compañías, de todos los tamaños, que aún no han experimentado la salida al exterior. También sería interesante la propuesta de un modelo dinámico que contemple los recursos y capacidades de las empresas y su puesta en marcha en entornos internacionales.

\section{Referencias}

Alonso, J., \& Donoso, V. (2000). Modelización del comportamiento de la empresa exportadora española. InformaciónComercial Española, 788, 35-58.

Andersson, S. (2000). The Internationalization of the Firm from an Entrepreneurial Perspective. International Studies of Management \& Marketing, 30(1), 63-92. http://dx.doi.org/10.1080/00208825.2000.11656783

Aragón, A., \& Monreal, J. (2008). La estrategia como factor de internacionalización de la pyme española. Revista Internacional de la Pequeña y Mediana Empresa, 1(1), 20-45.

Bilkey, W.J., \& Tesar, G. (1977). The Export Behavior of Smaller-Sized Wisconsin Manufacturing Firms. Journal of International Business Studies, 8(1), 93-98. http://dx.doi.org/10.1057/palgrave.jibs.8490783 
Brouthers, L.E., \& Xu, K. (2002). Product stereotypes strategy and performance satisfaction: The case of Chinese exporters. Journal of International Business Studies, 33, 657-677. http://dx.doi.org/10.1057/palgrave.jibs.8491038

Brouthers, L.E., \& Nakos, G. (2005). The role of systematic international market selection on small firms’ export performance. Journal of Small Business Management, 43, 363-381. http://dx.doi.org/10.1111/j.1540-627X.2005.00142.x

Calantone, R., \& Knight, G. (2000). The critical role of product quality in the international performance of industrial firms. Industrial Marketing Management, 29(6), 493-506. http://dx.doi.org/10.1016/S0019-8501(00)00124-3

Cavusgil, S.T. (1983). Success Factors in Export Marketing: An Empirical Analysis. Journal of International Marketing and Marketing Research, 8(2), 63-73.

Cavusgil, S.T., \& Nevin, J.R. (1981). International Determinant of Export Marketing Behaviour: An Empirical Investigation. Journal of Marketing Research, 18(1), 114-119. http://dx.doi.org/10.2307/3151322

Cavusgil, S.T., \& Zou, S. (1994). Marketing Strategy-Performance Relationship: An Investigation of the Empirical Link in Export Market Ventures. Journal of Marketing, 58(1), 1-21. http://dx.doi.org/10.2307/1252247

Claver Cortés, E., Rienda García, L., \& Quer Ramón, D. (2006). El comportamiento de las empresas familiares y no familiares en los mercados extranjeros: Un estudio comparativo. Revista Cuadernos de Gestión, 6(2), 11-27.

Dhanaraj, C., \& Beamish, P.W. (2003). A resource-based approach to the study of export performance. Journal of Small Business Management, 41, 242-261. http://dx.doi.org/10.1111/1540-627X.00080

Dunning, J.H. (1988). The Eclectic Paradigm of International Production: A Restatement and Possible Extension. Journal of International Business Studies, 19(1), 1-31. http://dx.doi.org/10.1057/palgrave.jibs.8490372

Dunning, J.H. (2001). The Eclectic (OLI) Paradigm of International Production: Past, Present and Future. International Journal of the Economics of Business, 8(2), 173-190. http://dx.doi.org/10.1080/13571510110051441

Escudero-Torres, M. ${ }^{a}$ A., Hurtado-Torres, N.E., De La Torre-Ruiz, J.M., \& Aguilera-Caracuel, J. (2011). La internacionalización de la empresa como fuente generadora de innovación: Una aplicación empírica al sector alimentario. EsicMarket, 139, 51-75. 
Estrella Ramón, A.M., Jiménez Castillo, D., Ruiz Leal, J.L., \& Sánchez Pérez, M. (2012). ¿Cómo compiten las pymes en los mercados internacionales? Análisis de un clúster local con vocación exportadora. Investigaciones europeas de Dirección y Economía de la Empresa, 18(1), 87-99. http://dx.doi.org/10.1016/S1135-2523(12)60062-8

Fernández Moreno, M.V., Peña García-Pardo, I., \& Hernández Perlines, F. (2007). Factores determinantes del éxito exportador. El papel de la estrategia exportadora en las empresas de economía social. En J.C. Ayala Calvo (Ed.). Conocimiento, innovación y emprendedores: Camino al futuro. Universidad de La Rioja Grupo de Investigación FEDRA.

Flor Peris, M.L., \& Oltra Mestre, M.J. (2010). La estrategia exportadora de la empresa y su relación con el resultado internacional. Investigaciones Europeas de Dirección y Economía de la Empresa, 16(1), 15-29. http://dx.doi.org/10.1016/S1135-2523(12)60001-X

García-Canal, E. (2004). El papel de las alianzas estratégicas en la internacionalización de las empresas españolas. Universia Business Review, 3(3), 70-83.

Gómez, M., \& Valenzuela, A. (2005). Export Marketing Strategies for High Performance: Evidence from Spanish Exporting Companies. Journal of Euro-Marketing, 15(1), 5-28. http://dx.doi.org/10.1300/J037v15n01_02

Graves, C., \& Thomas, J. (2006). Internationalization of Australian family businesses: A managerial capabilities perspective. Family Business Review, 19(3), 207-224. http://dx.doi.org/10.1111/j.17416248.2006.00066.x

Hair, J., Anderson, R., Tatham, R., \& Black, W. (1999). Análisis Multivariante. $5^{a}$ edición. Madrid: Prentice Hall Ediciones.

Horská, E (2011). European Studies on Intercultural dimension of International Business. Nitra: Slovak University of Agriculture in Nitra. R. Araque, M.J. Montero \& B. Gutiérrez, Managing international business: Case of Spain (Chapter 6, pp. 109-116).

Johanson, J., \& Vahlne, J.E. (1977). The internationalization process of the firm-a model of knowledge development and increasing foreign market commitments. Journal of International Business Studies, 8(1), 25-34. http://dx.doi.org/10.1057/palgrave.jibs.8490676

Johanson, J., \& Vahlne, J.E. (1990). The Mechanism of Internationalization. International Marketing Review, 7(4), 11-24. http://dx.doi.org/10.1108/02651339010137414

Johanson, J., \& Wiedersheim-Paul, F. (1975). The internationalization of the firm - four swedish cases. Journal of Management Studies, 12(3), 305-322. http://dx.doi.org/10.1111/j.1467-6486.1975.tb00514.x 
Kaleka, A., \& Katsikeas, S. (1995). Exporting problems: The relevance of export development. Journal of Marketing Management, 11, 499-515. http://dx.doi.org/10.1080/0267257X.1995.9964361

Katsikeas, C., Piercy, N.F., \& Ioannidis, C. (1996). Determinants of Export Perfomance in a European Context. European Journal of Marketing, 30(6), 6-35. http://dx.doi.org/10.1108/03090569610121656

Lages, L.F., Abrantes, J.L., \& Lages, C.R. (2008). The Stratadapt Scale: A Measure of Marketing Strategy Adaptation to International Business Markets. International Marketing Review, 25(5), 584-600. http://dx.doi.org/10.1108/02651330810904107

Leonidou, L., Katsikeas, C., \& Piercy, N. (1998). Identifying Managerial Influences on Exporting: Past Research and Future Directions. Journal of International Marketing, 6(2), 74-102.

Liberman, S., Baena, V., \& Moreno, F. (2010). La expansión internacional de la industria vitivinícola chilena. Estudio empírico de los factores y nivel exportador. Cuadernos de Gestión, 10(1), 35-74. http://dx.doi.org/10.5295/cdg.090174sl

Losada, F., Navarro, A., Ruzo, E., \& Barreiro J.M. (2006). La performance de exportación: Revisión teórica y propuesta de un marco integrador. Investigaciones Europeas de Dirección y Economía de la Empresa, 12(1), 85-106.

Martínez-Villavicencio, J. (2011). Factores que inciden en el desempeño exportador de las Pymes: Una aplicación empírica. EsicMarket, 140, 211-233.

Moini, A.H. (1995). An Inquiry into Successful Exporting: An Empirical Investigation Using a ThreeStage Model. Journal of Small Business Management, 33(3), 9-25.

Morgan, N., Kaleka, A., \& Katsikeas, C. (2004). Antecedents of Export Venture Performance: A Theoretical Model and empirical Assessment. Journal of Marketing, 68, 90-108. http://dx.doi.org/10.1509/jmkg.68.1.90.24028

Morgan, N.A., Vorhies, D.W., \& Mason, C.H. (2009). Market Orientation, Marketing Capabilities, and Firm Performance. Strategic Management Journal, 30(8), 909-920. http://dx.doi.org/10.1002/smj.764

Morgan, N.A., Katsikeas, C.S., \& Vorhies, D.W. (2012). Export Marketing Strategy Implementation, Export Marketing Capabilities, and Export Venture Performance. Journal of the Academy of Marketing Science, 40(2), 271-289. http://dx.doi.org/10.1007/s11747-011-0275-0

Navarro, A., Acedo, F.J., Robson, M.J., Ruzo, E., \& Losada, F. (2010). Antecedents and Consequences of Firms' Export Commitment: An Empirical Study. Journal of International Marketing, 18(3), 41-61. http://dx.doi.org/10.1509/jimk.18.3.41 
Navarro, A., Acedo, F.J., Losada, F., \& Ruzo, E. (2011). Integrated Model of Export Activity: Analysis of Heterogeneity in Managers' Orientations and Perceptions on Strategic Marketing Management in Foreign Markets. Journal of Marketing Theory and Practice, 19(2), 187-204.

http://dx.doi.org/10.2753/MTP1069-6679190205

Parada, P. (2012). Cómo preparar y aprovechar la expansión internacional. Harvard Deusto Business Review, 213, 4-14.

Piercy, N.F., Kaleka, A., \& Katsikeas, C.S. (1998). Sources of competitive advantage in high performing exporting companies. Journal of World Business, 33 (4), 378-393. http://dx.doi.org/10.1016/S10909516(99)80081-9

Pla-Barber, J., Villar-García, C., \& Escribá Esteve, A. (2010). La influencia de las características y percepciones directivas en los nuevos modelos de internacionalización. Economía industrial, 375, $101-112$.

Rastrollo Horrillo, M.A., Martín Armario, J., \& González Pachón, J.R. (2014). Claves de internacionalización de las empresas consultoras de ingeniería civil. Un caso de éxito: Grupo TYPSA. Intangible Capital, 10(3), 401-424. http://dx.doi.org/10.3926/ic.485

Reuber, R., \& Fischer, E. (1998). The Influence of the Management Team's International Experience on the Internationalization Behaviours of Smes. Journal of International Business Studies, 28(4), 807-825. http://dx.doi.org/10.1057/palgrave.jibs.8490120

Rialp, A., Rialp, J., \& Knight, G.A. (2005). The phenomenon of early internationalizing firms: What do we know after a decade (1993-2003) of scientific inquiry?. International Business Review, 14, 147-166. http://dx.doi.org/10.1016/j.ibusrev.2004.04.006

Rodríguez Serrano, Ma .A., Ruiz Moreno, C., \& Martín Armario, E. (2012). Las empresas Born Global: Un enfoque de capacidades dinámicas. Revista internacional de la Pequeña y Mediana Empresa, 1(4), 49-66.

Robertson, C., \& Chetty, S.K. (2000). A contingency-based approach to understanding export performance. International Business Review, 9, 211-235. http://dx.doi.org/10.1016/S0969-5931(99)00037-2

Sapienza, H.J., Autio, E., George, G., \& Zahra, S.A. (2006). A capabilities perspective on the effects of early internationalization on firm survival and growth. Academy of Management Review, 31(4), 914-933. http://dx.doi.org/10.5465/AMR.2006.22527465

Sousa, C.M., Martínez-López, F. J., \& Coelho, F. (2008). The determinants of export performance: A review of the research in the literature between 1998 and 2005. International Journal of Management Review, 10(4), 343-374. http://dx.doi.org/10.1111/j.1468-2370.2008.00232.x 
Sousa, C.M., \& Lengler, J. (2009). Psychic Distance, Marketing Strategy and Performance in Export Ventures of Brazilian Firms. Journal of Marketing Management, 25(5/6), 591-610. http://dx.doi.org/10.1362/026725709X461876

Tan, Q., \& Sousa, C.M. (2011). Research on Export Pricing: Still Moving Toward Maturity. Journal of International Marketing, 19(3), 1-35. http://dx.doi.org/10.1509/jimk.19.3.1

Villena Manzanares, F., \& Souto Pérez, J.E. (2015). El impacto de los intangibles estratégicos en el desempeño exportador de la PYME manufacturera. Intangible Capital, 11(1), 13-40. http://dx.doi.org/10.3926/ic.567

Wernerfelt, B. (1984). A resource-based view of the firm. Strategic Management Journal, 5, 171-180. http://dx.doi.org/10.1002/smj.4250050207

Yeoh, P.L., \& Jeong, I. (1995). Contingency relationship between entrepreneurship, export cannel structure and environment: A proposed conceptual model of export performance. European Journal of Marketing, 29(8), 95-115. http://dx.doi.org/10.1108/03090569510097574

Zou, S., Andrus, D.M., \& Norvell, D.W. (1997). Standardization of International Marketing Strategy by Firms from a Developing Country. International Marketing Review, 14(2), 107-123. http://dx.doi.org/10.1108/02651339710170203

Zou, S., Fang, E., \& Zhao, S. (2003). The effect of export marketing capabilities on export performance: an investigation of Chinese exporters. Journal of International Marketing, 11, 32-55. http://dx.doi.org/10.1509/jimk.11.4.32.20145

Intangible Capital, 2016 (www.intangiblecapital.org)

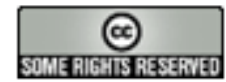

Article's contents are provided on an Attribution-Non Commercial 3.0 Creative commons license. Readers are allowed to copy, distribute and communicate article's contents, provided the author's and Intangible Capital's names are included. It must not be used for commercial purposes. To see the complete license contents, please visit http://creativecommons.org/licenses/by-nc/3.0/. 